\title{
Second-wave Dynamics of COVID-19: Impact of Behavioral Changes, Immunity Loss, New Strains, and Vaccination
}

Alex De Visscher ( $\square$ alex.devisscher@concordia.ca )

Concordia University https://orcid.org/0000-0002-2464-7605

\section{Brian Sutton}

1Department of Chemical and Materials Engineering, Gina Cody School of Engineering and Computer Science, Concordia University, Montreal, Quebec, Canada

\section{Tom Sutton}

1Department of Chemical and Materials Engineering, Gina Cody School of Engineering and Computer Science, Concordia University, Montreal, Quebec, Canada

\section{Research Article}

Keywords: SARS-CoV-2, Herd Immunity, Social Distancing, Mutations, Variants, NPI

Posted Date: February 22nd, 2021

DOI: https://doi.org/10.21203/rs.3.rs-195879/v1

License: (c) (i) This work is licensed under a Creative Commons Attribution 4.0 International License. Read Full License 
Second-wave dynamics of COVID-19: Impact of behavioral changes, immunity loss, new strains, and vaccination

\author{
Alex De Visscher ${ }^{*}$, Brian Sutton ${ }^{2}$, Tom Sutton ${ }^{3}$ \\ ${ }^{1}$ Department of Chemical and Materials Engineering, Gina Cody School of Engineering and \\ Computer Science, Concordia University, Montreal, Quebec, Canada. Tel.: +1-514-848-2424 \\ ext. 3488 . \\ E-mail: alex.devisscher@concordia.ca \\ ORCID: 0000-0002-2464-7605 \\ ${ }^{2}$ Glasgow, U.K. \\ ${ }^{3}$ Cambridge, U.K. \\ *Corresponding author. e-mail: alex.devisscher@concordia.ca
}




\begin{abstract}
An epidemiological model for COVID-19 developed earlier was extended to determine the effects of behavioral changes, immunity loss, and vaccination on second and subsequent wave dynamics of the pandemic. A model variant that distinguishes four demographic groups with different infection rates and mortality rates was developed to test the hypothesis that behavioral divergence between groups can explain both the larger incidence and lower mortality rate of COVID-19's second wave. A model version that incorporates immunity loss was developed to test the hypothesis that immunity loss can explain the second wave. Simulations indicate that of the two hypotheses, only the former is consistent with observed trends. Nevertheless, loss of immunity can significantly increase total number of deaths in the long run, particularly in cases where vaccine distribution is barely sufficient to reach herd immunity. The observed trends are illustrated with detailed simulations of the progression of COVID-19 in the United Kingdom, including the appearance of new strains. The U.K. case study indicates the extent to which NPI can be relaxed during the distribution of the vaccine.
\end{abstract}

Keywords: SARS-CoV-2; Herd Immunity; Social Distancing; Mutations; Variants; NPI

\title{
Declarations
}

Funding: none

Conflicts of interest/Competing interests: none

Availability of data and material: upon request to the author 
Code availability: upon request to the author

\section{Introduction}

COVID-19 is a respiratory disease first observed in Wuhan, China, in late 2019. It is caused by the SARS-CoV-2 virus. It is similar to other coronaviruses such as the original SARS virus. The virus spread to Europe and North America in early 2020 and was declared a pandemic by the World Health Organization on March 11, 2020. After an initial peak of daily deaths in April 2020, the death rate declined in the countries first affected but the virus spread to the rest of the world, causing a broad peak of daily deaths in the late summer of 2020.

The widespread use of non-pharmaceutical interventions (NPIs) such as lockdowns, wearing of facemasks, frequent disinfection, and closure of nonessential services led to a retreat of the epidemic in most countries but the easing of these interventions in late summer and early fall 2020 caused a resurgence of the disease in most countries, which persisted to early 2021. Globally, the "second wave" of fall 2020 was characterized by a vastly increased number of new cases per day, as well as increased number of new deaths per day, in comparison with the first wave of spring 2020, but with a lower case-mortality rate in the second wave in comparison with the first wave. Various factors could be responsible for the latter observation, including a possible decline of the mortality rate of the disease, better clinical procedures, a shift to a younger and less fragile segment of the population as the main carriers of the virus, and increased testing. Because of the significant number of asymptomatic cases, inadequate testing leads to underestimation of the number of cases, and hence higher case-mortality rates. 
A number of reports point at a gradual loss of immunity over a time span of several months after recovering from COVID-19. Edridge et al. [1] investigated reinfection from four seasonal human coronaviruses and found that reinfection is common after 12 months for all four viruses. Based on this observation, they anticipate that immunity for SARS-CoV-2 is short-lived. Immunity to SARS-CoV-2 is still poorly understood but based on [2,3], a duration of 5 months is a reasonable assumption at this time.

Many mutations of SARS-CoV-2 have been reported [4]. Most of these mutations have not been reported to be physiologically significant.

An early mutation that has been associated with increased infectivity is known as the D614G mutation. Korber et al. [5] observed that mutated virus copies systematically became more prevalent than the pre-mutation variant in a large number of countries and cities. This may explain why, for instance, the initial spread of COVID-19 was more gradual on the NorthAmerican West Coast, where the original variant took hold first, than on the North-American East Coast, where the mutated variant took hold from the beginning. There is no evidence that the D614G mutation leads to more severe infections [6].

A mutation of particular concern, VOC 202012/01, was found in southeast England in late 2020. Davies et al. [7] estimated this strain to be $56 \%$ more transmissible than pre-existing variants (confidence interval 50-74 \%).

Modeling the spread of an infectious disease is usually conducted by models based on the SIR (Susceptible-Infected-Recovered) model of Kermack and McKendrick [8]. A review of preCOVID models is given by Chowell et al. [9]. Early post-COVID models are by Lin et al. [10] and Roosa et al. [11]. De Visscher [12] used an extension of the SIR model to study the effect of NPIs on the expected mortality of the disease and applied the model to project future deaths from 
past deaths in Italy, France, and Iran. Huang and Qi [13] used a similar model to evaluate the second wave effect of an easing of NPIs. Wang et al. [14] developed a model that accounts for asymptomatic spread of COVID-19 and found that continued control is needed after a lockdown is eased. Kwuimy et al. [15] developed a similar model to evaluate the effect of governmentbased NPI initiatives and the behavioral response of the population. Zlatić et al. [16] developed a model that distinguishes detected and undetected cases in the infected population. Assuming that detected individuals would adjust their behavior to avoid spreading, it was shown that effective early testing can prevent an epidemic from growing explosively. Endo [17] argued, based on modeling results, that an effective way to reduce the spread of the virus is to focus on relatively rare superspreaders or superspreading events.

A much more sophisticated model was developed by Ferguson et al. [18]. This model calculates the spread of the disease within as well as outside family units in a stochastic manner. It also accounts for geographic spreading. The model is based on a similar model for influenza [19]. Based on comparisons with influenza pandemics such as the 1919-1920 "Spanish flu” pandemic, a second wave in the fall of 2020 was anticipated but the severity of the second wave in terms of the number of new cases was unexpectedly high. Factors that may have contributed to the severity include the reopening of schools after the summer vacation as well as NPI "fatigue", where many people relax on the precautions due to a perceived lowering of the risk. The first objective of this study is to test if the reopening of schools can explain the severity of the second wave. Second, when some degree of "herd immunity" (lowering of the reproduction rate of the disease resulting from partial immunity of the population) is established, a gradual loss of immunity can cause a resurgence of the disease. Our second objective was to test if this process can explain the occurrence of a second wave. 
Pfizer developed a vaccine against COVID-19 in late 2020. In most countries, elderly people and other at-risk segments of the population are vaccinated first to minimize the loss of life.

However, this choice may not halt the disease because elderly and at-risk people are not the main spreaders of the disease. An alternative strategy would be to vaccinate the main spreaders first to stop the epidemic in its tracks. Despite the low mortality of COVID among the active segment of the population, it is possible that this strategy would save more lives. The third objective of this paper is to test this hypothesis.

The distribution of vaccinations will alter the dynamic of the disease but this effect may be temporary due to the loss of immunity over time. The fourth objective of this study is to evaluate the long-term effect of vaccination in the case of immunity loss.

This paper is concluded with a case study in the United Kingdom. Based on a detailed fitting, a NPI history of the U.K. is reconstructed and followed by projections based on vaccination and NPI relaxing scenarios. The objective of this case study is to evaluate the extent of NPI relaxing that can be afforded by the vaccine, despite the emergence of a more infective strain of the virus.

\section{Model description}

\subsection{Original model description}

The model variants used here are extensions of the model of De Visscher [12]. For a full description of the original model, see the original paper. The original model assumes that the disease runs through the population from an Uninfected (U) to an Infected (I), a Sick (S), and a Seriously Sick (SS) state. Infected people can improve to a Better (B) state either directly from 
the Sick state or from the Seriously Sick stare. The Seriously Sick state can also lead to Death (D). The Better state leads to Recovery (R).

Mathematically, the infection rate $(\mathrm{U} \rightarrow \mathrm{I})$ is given by:

$$
r_{1}=\left(k_{11} I+k_{12} S+k_{13} S S+k_{14} B\right) \frac{U}{P}
$$

where $P$ is the total population. The infection rate $r_{1}$ is in infections per day. The rate constants $k_{11}, \ldots$ are expressed in day $^{-1}$. In the general cases in this paper, $P$ is assumed to be 100 million, whereas in the U.K. case study, $P$ equals 67.8 million. The default value of $k_{11}$ in this study is 0.4 $\mathrm{day}^{-1}$ for the general cases, and $0.39 \mathrm{day}^{-1}$ for the U.K. case. The features of new strains will be discussed in Section 2.5. The value of $k_{11}$ is allowed to vary over time to express NPIs. For details see [12].

The other rate constants in eq. (1) are given by:

$$
\begin{aligned}
& k_{12}=\frac{k_{11}}{2} \\
& k_{13}=\frac{k_{11}}{3} \\
& k_{14}=0
\end{aligned}
$$

In other words, recovering patients are assumed to be uninfective. Other infection reactions and their rates are as follows, except where explicitly stated otherwise: 


$$
\begin{array}{lll}
\mathrm{I} \rightarrow \mathrm{S}: & r_{2}=k_{2} I & k_{2}=\ln 2 / 5.1 \mathrm{day}^{-1} \\
\mathrm{~S} \rightarrow \mathrm{SS}: & r_{3}=k_{3} S & k_{3}=k_{5} / 9 \\
\mathrm{SS} \rightarrow \mathrm{D}: & r_{4}=k_{4} S S & k_{4}=k_{6} \times 15 / 85 \\
\mathrm{~S} \rightarrow \mathrm{B}: & r_{5}=k_{5} S & k_{5}=\ln 2 / 3.5 \mathrm{day}^{-1} \\
\mathrm{SS} \rightarrow \mathrm{B}: & r_{6}=k_{6} S S & k_{6}=\ln 2 / 10 \mathrm{day}^{-1} \\
\mathrm{~B} \rightarrow \mathrm{R}: & r_{7}=k_{7} B & k_{7}=\ln 2 / 10 \mathrm{day}^{-1}
\end{array}
$$

The model is run with the following initial conditions unless stated otherwise:

$$
\begin{aligned}
& I=100 \\
& S=10 \\
& S S=1 \\
& D=0 \\
& B=0 \\
& R=0
\end{aligned}
$$

The same initial conditions are used but for a later date in the case of a second or third strain. In the case of the U.K. case study, all initial conditions are multiplied with a correction factor as part of the model fit to the reported number of cumulative deaths.

\subsection{Model extension 1: demographic groups}


In this model extension, the population is divided into four demographic groups, ${ }^{1} \mathrm{P},{ }^{2} \mathrm{P},{ }^{3} \mathrm{P}$, and ${ }^{4} \mathrm{P}$. The properties of each group are shown in Table 1 . The numbers were chosen to be consistent with an overall mortality rate of $1.5 \%$ as used in the original model. Onder et al. [20] reported case mortality rates as low as $0.2 \%$ for age groups under forty, whereas the rates ranged from $8 \%$ to $20 \%$ for ages over 70 , depending on location and age group. Because underreporting of cases probably inflated these numbers, the lower end, $8 \%$, was used as the mortality rate for the old/at risk group in this model extension.

Table 1. Properties of the four assumed demographic groups: fraction of total population, fraction reaching Seriously Sick state $\left(f_{\mathrm{SS}}\right)$, mortality rate $\left(f_{\text {mort }}\right)$

\begin{tabular}{|c|c|c|c|c|}
\hline Group & Description & Fraction & $\boldsymbol{f}_{\text {SS }}$ & $f_{\text {mort }}$ \\
\hline${ }^{1} \mathrm{P}$ & active & $30 \%$ & $4 \%$ & $0.2 \%$ \\
\hline${ }^{2} \mathrm{P}$ & middle-aged & $40 \%$ & $10 \%$ & $1.5 \%$ \\
\hline${ }^{3} \mathrm{P}$ & old/at risk & $10 \%$ & $20 \%$ & $8 \%$ \\
\hline${ }^{4} \mathrm{P}$ & school-going & $20 \%$ & $4 \%$ & $0.2 \%$ \\
\hline
\end{tabular}

It can be demonstrated that the properties of the demographic groups relate to their rate constants $k_{3}, k_{4}, k_{5}$, and $k_{6}$ as follows:

$k_{3}=k_{5} \frac{f_{\mathrm{SS}}}{1-f_{\mathrm{SS}}}$ 


$$
k_{4}=k_{6} \frac{f_{\text {mort }}}{f_{\mathrm{SS}}-f_{\text {mort }}}
$$

The infection rates in this model are split into infection rates for each demographic group, where any demographic group can be infected by any other demographic group as well as its own:

$$
{ }^{j} r_{1}=\sum_{i=1}^{4}\left({ }^{i, j} k_{11}{ }^{i} I+{ }^{i, j} k_{12}{ }^{i} S+{ }^{i, j} k_{13}{ }^{i} S S+{ }^{i, j} k_{14}{ }^{i} B\right) \frac{{ }^{j} U}{P}
$$

To calculate the infection rates of four demographic groups, all 16 possible infection rate constants need to be known for each of $k_{11}, k_{12}$, etc. They were calculated as follows:

$$
{ }^{i, j} k_{11}=\sqrt{{ }^{i, i} k_{11} \cdot{ }^{j, j} k_{11}}
$$

for $k_{11}$, etc.

The model is set up in such a way that the efficiency of each NPI can be different for each demographic group.

\subsection{Model extension 2: immunity loss}

Immunity loss is modeled by assuming an additional transition:

$\mathrm{R} \rightarrow \mathrm{U}$ 
with a transition rate:

$r_{\mathrm{i}}=k_{\mathrm{i}} R$

For $k_{\mathrm{i}}$, values of $\ln (2) / 180$ and $\ln (2) / 90$ day $^{-1}$ were tested, representing immunity half-lives of 6 months and 3 months.

2.4. Model extension 3: vaccination

To represent vaccination, a new state, $\mathrm{V}$, is introduced. Vaccination is represented by the following transition:

$\mathrm{U} \rightarrow \mathrm{V}$

with a vaccination rate constant $k_{\text {vaccine }}$ expressed in vaccinations per day. The vaccination rate $r_{\text {vaccine }}$ is given by:

$$
r_{\text {vacine }}=f_{\text {vaccine }} \cdot k_{\text {vaccine }} \cdot \frac{U}{P}
$$

In eq. (18), the factor $f_{\text {vaccine }}$ is the effectiveness of the vaccine, assumed to be $95 \%$. The factor $U / P$ expresses the fact that it is not generally known who is immune and who is not, so a fraction of the vaccines is given to people who are already immune. 
The vaccination rate constant $k_{\text {vaccine }}$ as well as the effectiveness factor $f_{\text {vaccine }}$ can vary over time.

\subsection{Model extension 4: multiple COVID strains}

Multiple COVID strains need to be accounted for if they have different properties (infection rate, mortality). Approaches of multistrain epidemic dynamics are reviewed by Martcheva [21]. In the current simulation, it is assumed that the strains differ only by their infection rates. For the new strain that emerged in the U.K. in September 2020, an infection rate $70 \%$ higher than the infection rate of the original strain $\left(0.39 \mathrm{day}^{-1}\right)$ was assumed, at the higher end of the confidence interval of Davies et al. [7].

To distinguish infections with two different strains, two different infected states are distinguished: e.g., $\mathrm{I}_{1}$ and $\mathrm{I}_{2}$. Each state progresses in parallel, e.g., $\mathrm{I}_{1}$ to $\mathrm{S}_{1}$ to $\mathrm{SS}_{1}$, etc.

Strain 1 is assumed to be present from the beginning, Strain 2 is introduced in the model on simulation day September $22^{\text {nd }}, 2020$. A third strain with infection rate $100 \%$ higher than for Strain 1 was introduced in the model on January 15.

\section{Results}

3.1. Second wave dynamics: effect of changing infection rates

In general, infection rates are calculated with the following equation:

$k_{11}=k_{11,0}\left(1-f_{\mathrm{NPI}}\right)$ 
where $f_{\mathrm{NPI}}$ is the effectiveness of the NPI. In this model, the simulation is run for 450 days, representing March 2020-June 2021 for a typical country. The effectiveness values for the various demographic groups used in the simulation are shown in Table 2. Different scenarios were tested until a combination was found that led to an epidemic progression representative for European countries.

Table 2. Effectiveness of NPI, $f_{\mathrm{NPI}}$, for each demographic group (group $1=$ active; 2 = middleaged, 3 = old/at risk; 4 = school-going). Scenario 1: second wave due to behavioral/NPI change

\begin{tabular}{|l|l|l|l|l|l|}
\hline NPI \# & 1 & 2 & 3 & 4 & 5 \\
\hline Start day & 30 & 90 & 180 & 240 & 300 \\
\hline$f_{\text {NPI group 1 }}$ & 0.9 & 0.6 & 0.3 & 0.6 & 0.3 \\
\hline$f_{\text {NPI group 2 }}$ & 0.9 & 0.9 & 0.99 & 0.99 & 0.99 \\
\hline$f_{\text {NPI group 3 }} 0.9$ & 0.95 & 0.995 & 0.995 & 0.995 \\
\hline$f_{\text {NPI group 4 }} 0.9$ & 0.6 & 0 & 0.3 & 0 \\
\hline
\end{tabular}

The results of the simulation are shown in Figure 1. 


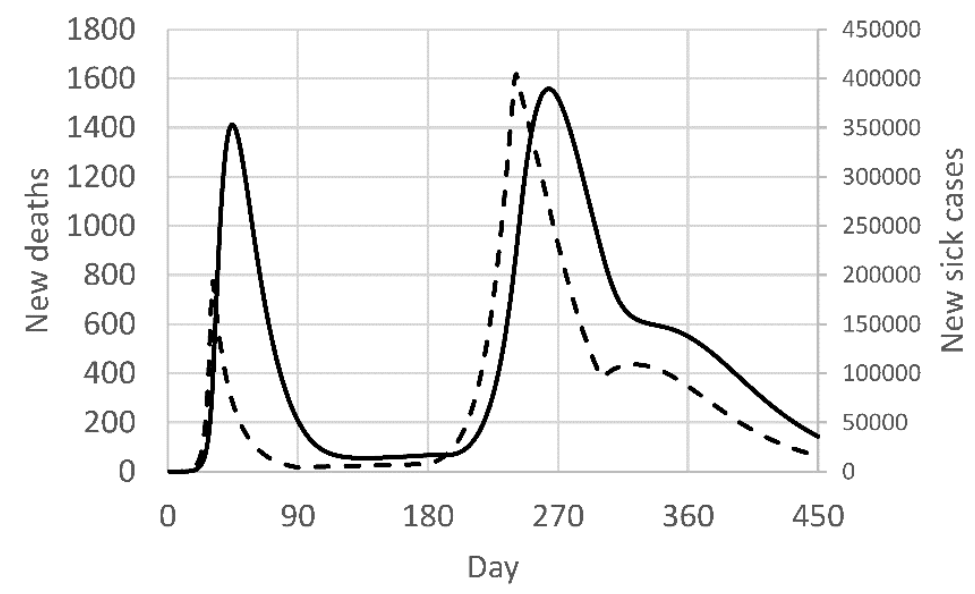

Figure 1. Daily new deaths (solid line, left axis) and new sick cases(dashed line, right axis) predicted by the model (extension 1) with the parameters from Table 2

In the simulation, the first wave peaks at 1400 daily deaths and 200,000 daily new sick cases, for a total population of 100 million. The daily new cases is much higher than actually observed in countries of a similar size because not all cases are identified or reported. Based on simulations for the previous study [12], countries underreport by about a factor 2-10 (data not shown) indicating that the actual reporting can be expected to be on the order of 20,000-100,000 new cases per 100 million, in line with what was actually observed during the first wave.

During the second wave, the daily new deaths peaks at about 1600, similar to the first wave, but the number of new sick cases doubles to about 400,000 (i.e., 40,000-200,000 actually reported). This means that whereas the incidence of the disease is much higher in the second wave, the mortality rate is about half in the second wave in comparison with the first wave. This is in line with actual observations, although the decline in case mortality rate was generally more pronounced in reality. This may have been due to increased testing during the second wave. To obtain the trends observed in Figure 1, it was necessary to assume a pronounced change in NPI effectiveness in both Group 1 (active) and Group 4 (school-going). Limiting the change to 
one of the two groups led to a much more slowly developing second wave with a lower peak. This indicates that the second wave cannot be attributed to a single demographic group but was the result of broad demographic behavioral changes, representing at least two of the four demographic groups.

To obtain a pronounced drop in the case mortality rate during the second wave, it was necessary to assume very high effectiveness of NPI in the middle-aged and old/at risk groups (99\% and above). These may not be realistic numbers. These high efficiencies were needed to keep cross infection between the more active and less active groups to levels where there is a significant divergence of the number of cases in the groups. This suggests that eq. (14) (for geometric averaging of infection rates) overestimates the actual cross infection rates. In practice, this would mean that there is less mingling between demographic groups than within. This confirms a finding of Klepac et al. [22] that social mixing is fairly stratified by age.

The cumulative number of deaths after 450 days in the model is 216,707 .

\subsection{Second wave dynamics: effect of lost immunity}

To evaluate if a second wave can be caused by declining immunity, a base case simulation is run with zero loss of immunity, with a NPI regime chosen so that the daily new deaths remain approximately constant over a prolonged period of time. The parameters are shown in Table 3.

Table 3. Effectiveness of NPI, $f_{\mathrm{NPI}}$, for each demographic group (group $1=$ active; 2 = middleaged, 3 = old/at risk; 4 = school-going); scenario 2 : second wave by immunity loss only 


\begin{tabular}{|l|l|l|}
\hline NPI \# & 1 & 2 \\
\hline Start day & 30 & 90 \\
\hline$f_{\text {NPI group 1 }}$ & 0.9 & 0.64 \\
\hline$f_{\text {NPI group 2 }}$ & 0.9 & 0.9 \\
\hline$f_{\text {NPI group 3 }}$ & 0.9 & 0.95 \\
\hline$f_{\text {NPI group 4 }}$ & 0.9 & 0.6 \\
\hline
\end{tabular}

The result of the calculations is shown in Figure 3. It is clear from the figure that immunity loss leads to a very gradual increase of the daily deaths over prolonged time, very unlike the dynamics of a second wave, with dramatic increases in death rates within a few weeks. It follows that loss of immunity is not a major contributor to second-wave dynamics of COVID-19.

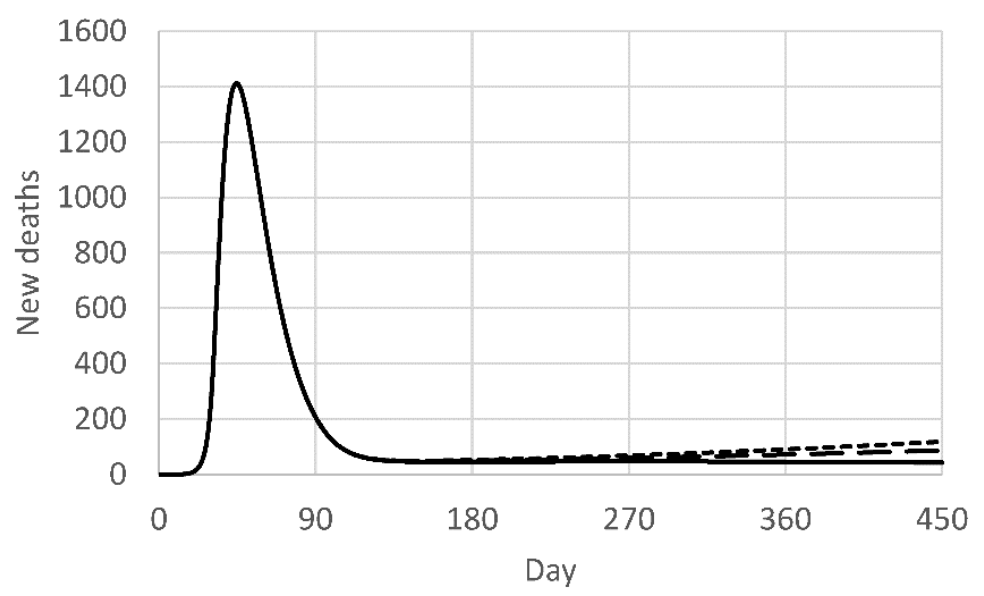


Figure 2. Effect of immunity loss on the onset of a second wave in a COVID-19 epidemic. Solid line: no loss of immunity; long dash: immunity disappears with a half-life of 6 months; short dash: immunity disappears with a half-life of 3 months.

In the base case (no immunity loss), the death rate declines to 46 per day on day 161, increases to 47.4 deaths per day on day 271 , and declines again to 42.9 on day 450 . When the immunity disappears with a half-life of six months, the minimum death rate is 48.1 per day on day 151 and increases gradually to 87.1 deaths per day on day 450 . When the half-life of immunity is 3 months, the minimum death rate is 49.6 on day 147 and increases to 118.6 deaths per day on day 450.

\subsection{Vaccination: effect of prioritizing demographic groups}

In this section we will evaluate which sequence of vaccination priorities leads to the least number of lives lost. In this section the simulations were run for 800 days to avoid missing slow trends. It is assumed that immunity is not lost. In practice, loss of immunity can be compensated by increased vaccination rates.

In this scenario, it is assumed that vaccination is accompanied by further loosening of NPIs. The sequence of NPI efficiencies is shown in Table 4.

Table 4 . Effectiveness of NPI, $f_{\mathrm{NPI}}$, for each demographic group (group $1=$ active; 2 = middleaged, 3 = old/at risk; 4 = school-going). Scenarios 3 and 4 : vaccination 


\begin{tabular}{|l|l|l|l|l|l|l|l|}
\hline NPI \# & 1 & 2 & 3 & 4 & 5 & 6 & 7 \\
\hline Start day & 30 & 90 & 180 & 240 & 300 & 350 & 400 \\
\hline$f_{\text {NPI group 1 }}$ & 0.9 & 0.6 & 0.3 & 0.6 & 0.3 & 0.3 & 0.3 \\
\hline$f_{\text {NPI group 2 }}$ & 0.9 & 0.9 & 0.99 & 0.99 & 0.99 & 0.9 & 0.8 \\
\hline$f_{\text {NPI group 3 }}$ & 0.9 & 0.95 & 0.995 & 0.995 & 0.995 & 0.95 & 0.9 \\
\hline$f_{\text {NPI group 4 }}$ & 0.9 & 0.6 & 0 & 0.3 & 0 & 0 & 0 \\
\hline
\end{tabular}

It is assumed that vaccination starts on day 300 and occurs in two phases, each lasting 100 days. During each phase, 200,000 vaccines are administered daily, totalling 40 million vaccines. In each phase, one demographic group is prioritized, receiving $70 \%$ of the vaccines, whereas the other groups receive $10 \%$ each. Every combination is tested and the total number of deaths on day 800 is calculated. The result is shown in Table 5 .

Table 5. Number of deaths after 800 days; one demographic group is prioritized on days 300-400 (priority 1, left to right) and one group is prioritized on days 400-500 (priority 2, top to down)

\begin{tabular}{|l|l|l|l|l|}
\hline prior. 2 $\backslash$ prior. 1 & group 1 & group 2 & group 3 & group 4 \\
\hline group 1 & 265,320 & 290,310 & 286,510 & 257,210 \\
\hline group 2 & 264,350 & 294,050 & 286,890 & 259,070 \\
\hline
\end{tabular}




\begin{tabular}{|l|l|l|l|l|}
\hline group 3 & 264,250 & 289,590 & 295,070 & 259,140 \\
\hline group 4 & 261,910 & 289,710 & 285,940 & 260,660 \\
\hline
\end{tabular}

All prioritizations lead to a total number of deaths ranging from 257,210 to 295,070 . This is a surprisingly narrow spread given that the mortality rates of the groups range from $0.2 \%$ to $8 \%$. Not vaccinating at all would lead to 467,730 deaths, almost twice as much. Among the scenarios, the most favorable one is vaccinating school-going youth first, followed by vaccinating the active group. It is generally more favorable to switch priorities after 100 days, and the first priority has a greater impact on the overall result than the second priority. However, given the narrow spread of the results, none of these trends should be taken as absolute. It is likely that small changes in the assumptions underlying the calculations will reverse these conclusions. It is more reasonable to conclude that, as long as an adequate level of vaccination is reached, it is of less importance who is prioritized.

Figure 3 shows the daily deaths over time in the vaccination scenario where Group 4 is prioritized first and Group 3 next. This scenario is compared with a single vaccination phase, and no vaccination at all. It is clear that vaccination has a profound effect and has the potential to eradicate the epidemic, even with incomplete vaccination. This is because the scenario assumes sufficient NPIs are in place to ensure herd immunity is maintained. 


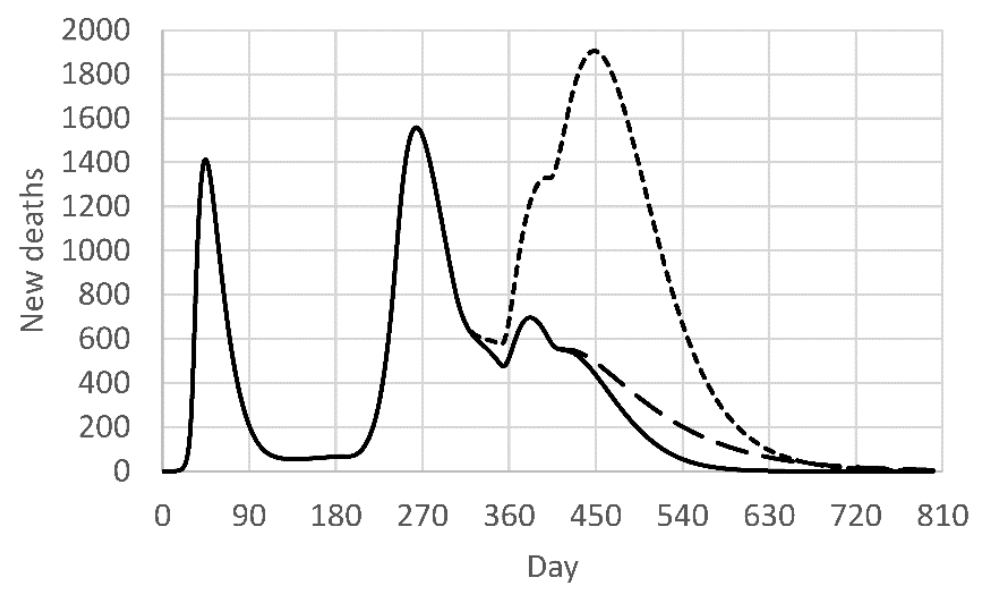

Figure 3. Daily deaths in three vaccination scenarios: school-going youth prioritized (day 300400) followed by active population prioritized (day 400-500) (solid line); only school-going youth prioritized (day 300-400) (long dash); no vaccination (short dash).

\subsection{Vaccination: effect of loss of immunity}

In this section, the same scenarios will be repeated, but this time it is assumed that immunity is only temporary, with immunity half-lives of 6 months or 3 months. It is assumed that the rate of immunity loss is the same regardless of whether immunity was obtained by the disease or the vaccine. It is assumed that health authorities anticipate the loss of immunity by continuing the vaccination. During days 500-800 it is assumed that all demographic groups are prioritized according to their size. The results are shown in Figure 4. 


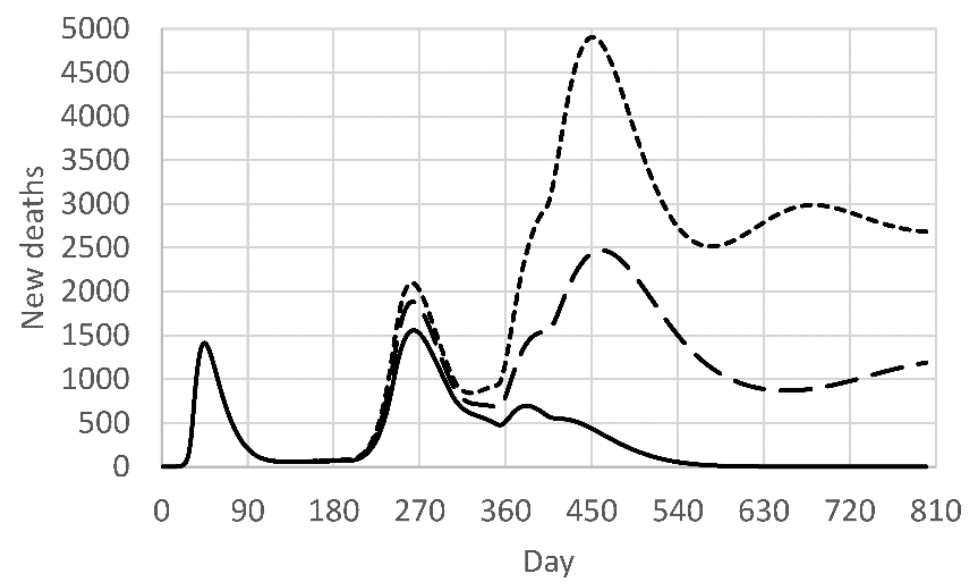

Figure 4. Daily deaths in continuing vaccination scenario (200,000 vaccines per day): no loss of immunity (solid line); immunity half-life 6 months (day 300-400) (long dash); immunity half-life 3 months (short dash).

In the absence of loss of immunity, the total number of deaths is 257,210 in 800 days. When the half-life of immunity is 6 months, this number is 821,830 , with a half-life of 3 months, the death toll is as high as $1,594,800$. This profound effect is because 200,000 vaccinations per day, $0.2 \%$ of the total population per day, is barely enough to achieve herd immunity.

The loss of immunity can be compensated by administering more vaccines. This is demonstrated in Figure 5, with 200,000, 600,000, and 1,200,000 vaccinations administered per day and an immunity half-life of 3 months. 


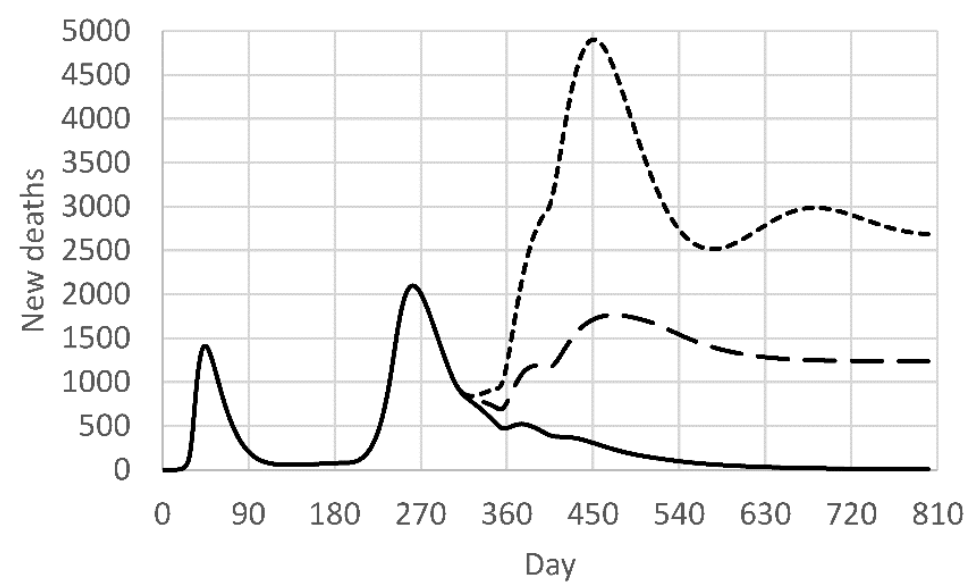

Figure 5. Daily deaths in continuing vaccination scenario, immunity half-life 3 months: 1,200,000 vaccines per day (solid line); 600,000 vaccines per day (long dash); 200,000 vaccines per day (short dash).

The death toll after 800 days is $1,594,800$ with 200,000 vaccinations per day, 832,190 deaths with 600,000 vaccinations per day, and 283,720 deaths with 1,200,000 vaccinations per day. The latter is similar to the number obtained in the absence of immunity loss. It is interesting to calculate the number of active immunizations administered over one half-life, accounting for the effectiveness of the vaccine in the latter case. That number is $0.95 \times 1,200,000 \times 90=102.6$ million. It follows that immunization is effective if a number of active immunizations equal to the entire population is administered every half-life. This is in the worst-case scenario where there is no information on who is immune and who is not. When vaccines are administered in a more organized manner, the same result should be achievable with fewer vaccinations.

\subsection{U.K. case study}


In this case study, actual COVID-19 daily death numbers were taken from Worldometer (https://www.worldometers.info/coronavirus/country/uk/) and the model variant with multiple COVID-19 strains was fitted to the data by adjusting the timing and effectiveness of consecutive NPI initiatives until a reasonable agreement between modeled and reported death data was obtained, up until the reporting date 28th January 2021. The NPI effectiveness values are given in Table 6, along with the dates they take effect. Because detailed disaggregation of deaths per day by age group is not publicly available, the entire population was modeled as a single demographic group, as in the original version of the model [12]. The model predicts that 2.7 million people in the U.K. had contracted COVID-19 by the end of June 2020. This corresponds well with an antibody study by Ward et al. [23] which led to an estimate of 3.36 million. Given that the actual COVID-19 death number is probably underreported, leading to an underestimated number of cases in the model, this is an excellent agreement.

As indicated above, a second strain with infection rate $70 \%$ higher than the original strain and a third strain with an infection rate $100 \%$ higher than the original strain (worst-case scenario) was introduced on September $22^{\text {nd }}$ and January $15^{\text {th }}$, respectively. In the base case scenario, the third strain does not gain a hold because of the high effectiveness of the NPI used in the model at that time $(97.5 \%)$. This high value was needed to predict recent deaths occurring with the new strain and may indicate that the infectiveness of the second strain (at $70 \%$ higher than the first) is overestimated in our model.

In the base case scenario, it is assumed that vaccination starts on January $1^{\text {st }}, 2021$ with 250,000 vaccinations per day, which is ramped up to 400,000 per day on February $1^{\text {st }}, 2021$, and thereafter. These numbers are conservative estimates. The vaccinations started on December $8^{\text {th }}$, 2020 in the U.K. but immunity takes three weeks to take effect. In the model, it is assumed that 
the effectiveness of the vaccines administered during the first month is $70 \%$, and that the effectiveness of the vaccines administered after the first month is $95 \%$. This allows for possible optimizations of the vaccination, partly owing to the variety of vaccines that are and will be available in the U.K..

The result of this scenario is shown in Figure 6 as the solid line. In the base scenario, the cumulative number of deaths after 800 days of simulation (i.e., on April $10^{\text {th }}, 2022$ ) is 128,436 . In scenario 2 , the same situation is simulated in the absence of vaccinations. The cumulative number of deaths is only slightly higher than in the base case, at 133,712 . The small difference is due to the fact that the assumed effectiveness is very high. To calculate the effect of loosening the NPIs after the start of the vaccination program, a decrease of the NPI effectiveness by $15 \%$ was introduced on March $7^{\text {th }}, 2021$, from $97.5 \%$ to $82.5 \%$. This date was chosen because it has been suggested as a potential reopening day for schools by the U.K. Government. This is scenario 3, which is also shown in Figure 6. This leads to a cumulative deaths number of 134,082, about the same as in the absence of a vaccine, but maintaining the high NPI effectiveness after March 7.

Finally, we calculated how this loosening of the NPIs would affect deaths in the absence of a vaccine. The result is off the scale in Figure 6. In total, loosening NPIs to a $15 \%$ decrease of the NPI effectiveness in the absence of a vaccine leads to 669,443 deaths by day 800 . Reported COVID-19 deaths are also included in the figure at weekly intervals. 


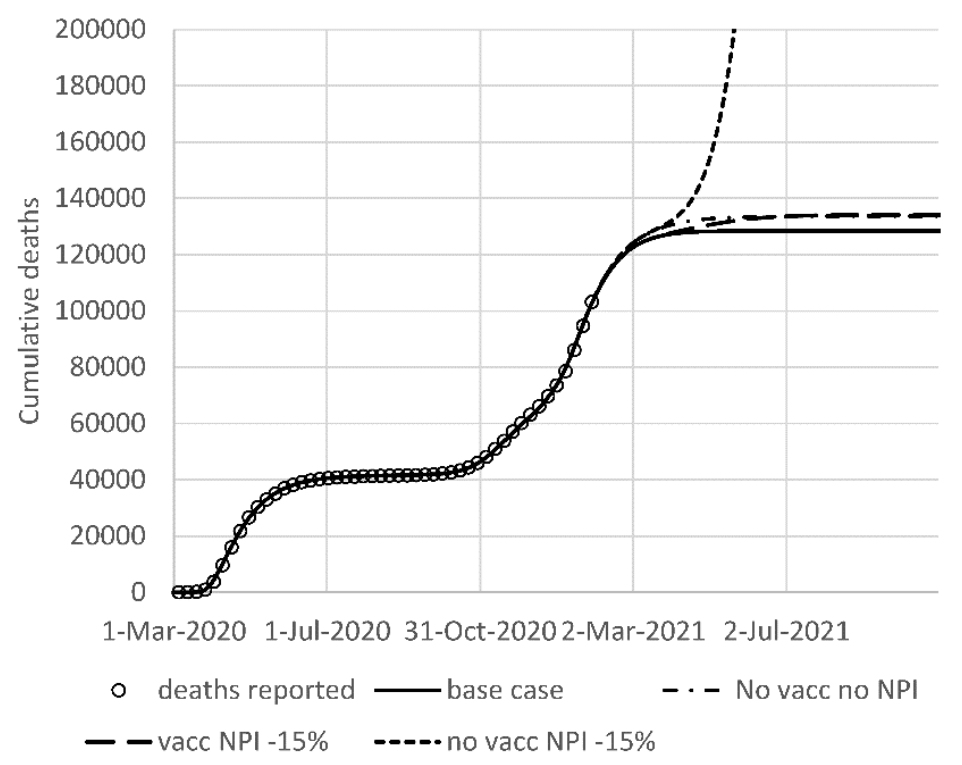

Figure 6. Cumulative deaths reported and modeled with vaccination, without loosening of NPIs (solid line); without vaccination, without loosening of NPIs (dot-dash line); with vaccination, with a $15 \%$ reduction of NPI effectiveness (long dash); and without vaccination, with a $15 \%$ reduction of NPI effectiveness (short dash).

Table 6. NPI effectiveness with dates they take effect.

\begin{tabular}{|l|l|l|l|}
\hline Date & Effectiveness & Date & Effectiveness \\
\hline Mar $23^{\text {rd }}, 2020$ & 0.843 & October $27^{\text {th }}, 2020$ & 0.805 \\
\hline May $15^{\text {th }}, 2020$ & 0.84 & November $6^{\text {th }}, 2020$ & 0.865 \\
\hline July $4^{\text {th }}, 2020$ & 0.8 & December $2^{\text {nd }}, 2020$ & 0.775 \\
\hline August $4^{\text {th }}, 2020$ & 0.74 & December $16^{\text {th }}, 2020$ & 0.875 \\
\hline August $15^{\text {th }}, 2020$ & 0.66 & December $23^{\text {rd }}, 2020$ & 0.775 \\
\hline
\end{tabular}




\begin{tabular}{|l|l|l|l|}
\hline September $1^{\text {st }}, 2020$ & 0.65 & December $28^{\text {th }}, 2020$ & 0.825 \\
\hline September $14^{\text {th }}, 2020$ & 0.635 & December $31^{\text {st }}, 2020$ & 0.775 \\
\hline September $24^{\text {th }}, 2020$ & 0.625 & January $3^{\text {rd }}, 2021$ & 0.975 \\
\hline October $5^{\text {th }}, 2020$ & 0.675 & March $7^{\text {th }}, 2021$ & 0.825 \\
\hline October $19^{\text {th }}, 2020$ & 0.745 & & \\
\hline
\end{tabular}

Figure 7 shows the same data set, but as daily deaths. In the figure, the reported data are weekly averages. In the model, when the entire population is modeled as a single demographic group, the number of seriously sick people is proportional to the daily new deaths due to eq. (7). The number of seriously sick can be calculated by multiplying the daily number of deaths by 81.75 .

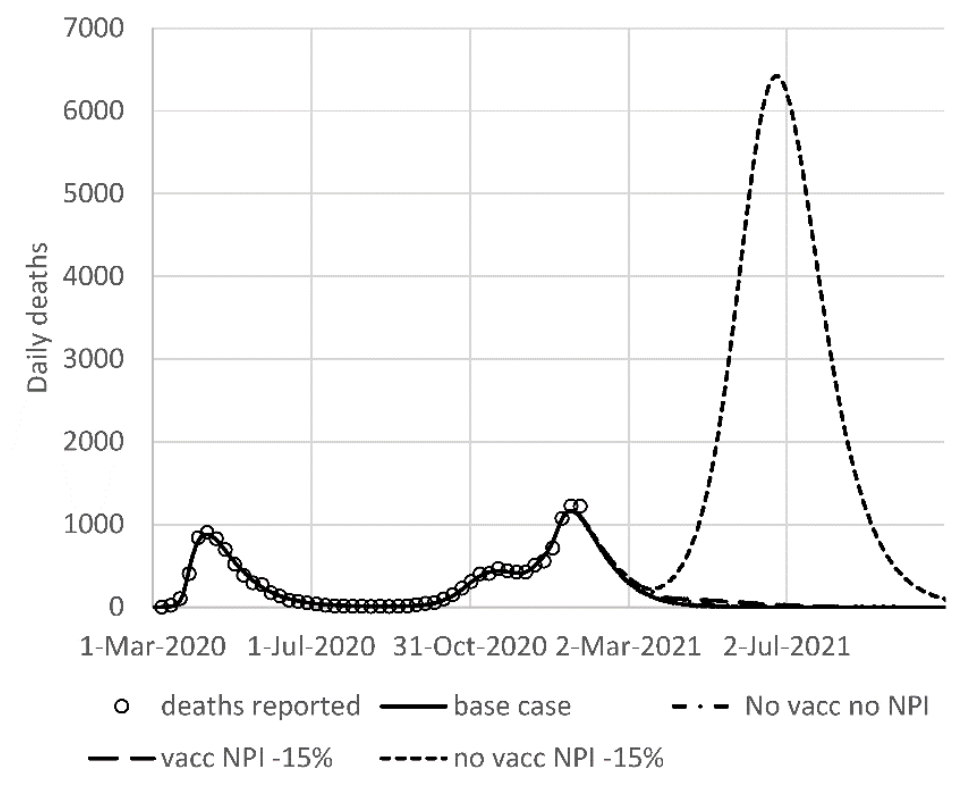


Figure 7. Daily deaths reported (weekly averages) and modeled with vaccination, without loosening of NPIs (solid line); without vaccination, without loosening of NPIs (dot-dash line); with vaccination, with a $15 \%$ reduction of NPI effectiveness (long dash); and without vaccination, with a $15 \%$ reduction of NPI effectiveness (short dash).

Figure 8 shows the dominance of the original (pre-September 2020, i.e., prior to the $2^{\text {nd }}$ variant) COVID-19 variants in the UK as modeled, with the four scenarios. It is clear from the figure that the original strains are rapidly declining in importance. This figure may somewhat overestimate the dominance of the new strains because a value of $70 \%$ higher infection rate was assumed for strain VOC 202012/01 that started in the U.K. in late 2020, which is on the upper end of the confidence interval estimated by Davies et al. [7].

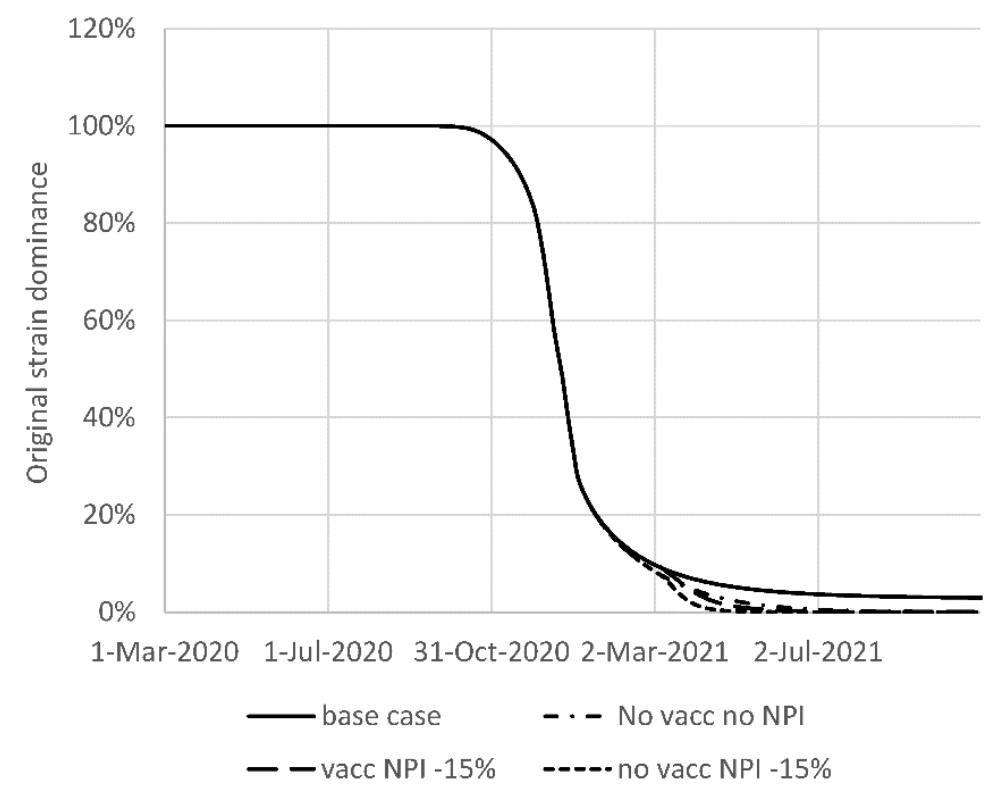

Figure 8. Dominance of the original (pre-September 2020) COVID-19 strains in the U.K. , as calculated by the model with the four scenarios from Figures 6 and 7 . 


\section{Conclusions}

An epidemiological model developed recently was extended in a number of ways to evaluate factors contributing to second-wave dynamics of COVID-19. It was shown that changes in the effectiveness of NPIs for younger age groups (labelled "active" and "school-going") comprising $50 \%$ of the population can explain both the second wave itself and the reduced mortality rate during the second wave. Loss of immunity affects the dynamics of COVID-19 too slowly to be a major factor in second-wave dynamics. However, loss of immunity has a pronounced long-term effect on the number of deaths resulting from COVID. The effect of prioritizing demographic groups for vaccination has a surprisingly small effect on the overall death number from the virus. In the scenarios run in this paper, vaccinating the most active segments of the population had the most favorable outcome, but this may be specific to the assumptions used in the model and may not bear out in actual practice.

A case study of the United Kingdom shows that the model can accurately describe death numbers due to COVID-19. This calibrated model predicts that vaccination in the U.K. at the current and planned rates will be adequate if maintained, and will enable a loosening of the NPIs, in this case on March 7th 2021 when it is thought U.K. schools might reopen, allowing the effectiveness of the NPIs to fall by $15 \%$.

\section{References}


[1] Edridge A.W.D., Kaczorowska J., Hoste A.C.R., Bakker M., Klein M., Loens K., Jebbink M.F., Matser A., Kinsella C.M., Rueda P., Ieven M., Goossens H., Prins M., Sastre P., Deijs M., van der Hoek L. (2020). Nature Med. 26, 1691-1693.

[2] Hall V., Foulkes S., Charlett A., Atti A., Monk E.J.M., Simmons R., Wellington E., Cole M.J., Saei A., Oguti B., Munro K., Wallace S., Kirwan P.D., Shroti M., Vusirikala A., Rokadiya S., Kall M., Zambon M., Ramsay M., Brooks T., Brown C.S., Chand M.A., Hopkins S. (2021). Do antibody positive healthcare workers have lower SARS-CoV-2 infection rates than antibody negative healthcare workers? Large multi-centre proepective cohort study (the SIREN study), England: June to November $2020 . \quad$ medRxiv https://www.medrxiv.org/content/10.1101/2021.01.13.21249642v1.

[3] Ledford H. (2021). Large study of UK health-care workers suggests that most people are immune for months after catching COVID-19 for the first time. Nature News 14 January 2021 https://doi.org/10.1038/d41586-021-00071-6.

[4] Coppée F., Lechien J.R., Declèves A.E., Tafforeau L., Saussez S. (2020). Severe acute respiratory syndrome coronavirus 2: Virus mutations in specific European populations. New Microbe. New Infect. 36, 100696.

[5] Korber B., Fischer W.M., Gnanakaran S., Yoon H., Theiler J., Abfalterer W., Hengartner N., Giorgi E., Bhattacharya T., Foley B., Hastle K.M., Parker M.D., Partridge D.G., Evans C.M., Freeman T.M., de Silva T.I., McDanal C., Perez L.G., Tang H., Moon-Walker A., Whelan S.P., LaBranche C.C., Saphire E.O., Montefiori D.C. (2020). Tracking changes in SARS-CoV-2 spike: Evidence that D614G increases infectivity of the COVID-19 virus. Cell 182, 812-827.

[6] Grubaugh N.D., Hanage W.P., Rasmussen A.L. (2020). Making sense of mutation: What D614G means for the COVID-19 pandemic remains unclear. Cell 182, 794-795. 
[7] Davies N., Barnard R.C., Jarvis C.I., Kucharski A.J., Munday J., Pearson C.A.B., Russell T.W., Tully D.C., Abbott S., Gimma A., Waites W., Wong K.L.M., van Zantvoort K., Eggo R.M., Funk S., Jit M., Atkins K.E., Edmunds J. (2020). Estimated transmissibility and severity of novel SARS-CoV-2 Variant of Concern 202012/01 in England. CMMID Repository, in review, https://cmmid.github.io/topics/covid19/uk-novel-variant.html.

[8] Kermack W.O., McKendrick A.G. (1927). A contribution to the mathematical theory of epidemics. Proc. R. Soc Edinburgh A 115, 700-721.

[9] Chowell G., Sattenspiel L., Bansal S., Viboud C. (2016). Mathematical models to characterize early epidemic growth: A review. Phys. Life Rev. 18, 66-97.

[10] Lin Q., Zhao S., Gao D., Lou Y., Yang S., Musa S.S., Wang M.H., Cai Y., Wang W., Yang L., He D. (2020). A conceptual model for the coronavirus disease 2019 (COVID-19) outbreak in Wuhan, China with individual reaction and governmental action. Int. J. Infect. Diseases 93, 211-216.

[11] Roosa K., Lee Y., Luo R., Kirpich A., Rothenburg R., Hyman J.M., Yan P., Chowell G. (2020). Real-time forecasts of the COVID-19 epidemic in China from February $5^{\text {th }}$ to February $24^{\text {th }}, 2020$. Infect. Disease Model. 5, 256-263.

[12] De Visscher A. (2020). The COVID-19 pandemic: Model-based evaluation of nonpharmaceutical interventions and prognoses. Nonlin. Dynamics 101, 1871-1887.

[13] Huang J, Qi G. (2020). Effects of control measures on the dynamics of COVID-19 and double-peak behavior in Spain. Nonlin. Dynamics 101, 1889-1899.

[14] Wang X., Wang S., Lan Y., Tao X., Xiao J. (2020). The impact of asymptomatic individuals on the strength of public health interventions to prevent the second outbreak of COVID-19. Nonlin. Dynamics 101, 2003-2012. 
[15] Kwuimy C.A.K., Nazari F., Jiao X., Rohani P., Nataraj C. (2020). Nonlinear dynamic analysis of an epidemiological model for COVID-19 including public behavior and government action. Nonlin. Dynamics 101, 1545-1559.

[16] Zlatić V., Barjašić I., Kadović A., Štefančić H., Gabrielli A. (2020). Bi-stability of SUDR+K model of epidemics and test kits applied to COVID-19. Nonlin. Dynamics 101, $1635-1642$.

[17] Endo A., Abbott S., Kucharski A.J., Funk S. (2020). Estimating the overdispersion in COVID-19 transmission using outbreak sizes outside China. Wellcome Open Research article 5-67 https://wellcomeopenresearch.org/articles/5-67.

[18] Ferguson N.M., Laydon D., Nedjati-Gilani G., Imai N., Ainslie K., Baguelin M., Bhatia S., Boonyasiri A., Cucunuba Z., Cuomo-Dannenburg G., Dighe A., Dorigatti I., Fu H., Gaythorpe K., Green W., Hamlet A., Hinsley W., Okell L.C., van Elsland S., Thompson H., Verity R., Volz E., Wang H., Wang Y., Walker P.G.T., Walters C., Winskill P., Whittaker C., Donnelly C.A., Riley S., Ghani A.C. (2020). Report 9: Impact of non-pharmaceutical interventions (NPIs) to reduce COVID-19 mortality and healthcare demand. Imperial College COVID-19 Response Team, DOI: https://doi.org/10.25561/77482.

[19] Ferguson N.M., Cummings D.A.T., Cauchemez S., Fraser C., Riley S., Meeyai A., Iamsirithaworn S, Burke D.S. (2005). Strategies for containing an emerging influenza pandemic in Southeast Asia. Nature 437, 209-214.

[20] Onder, G, Rezza G., Brusaferro S. (2020). Case fatality rate and characteristics of patients dying in relation to COVID-19 in Italy. J. Am. Med. Assoc. 323, 1775-1776.

[21] Martcheva M. (2015). An Introduction to Mathematical Epidemiology. Springer. 
[22] Klepac P., Kucharski A.J., Conlan A.J.K., Kissler S., Tang M.L., Fry H., Gog J.R. (2020).

Contacts in context: Large-scale setting-specific social mixing matrices from the BBC Pandemic project. medRxiv https://doi.org/10.1101/2020.02.16.20023754.

[23] Ward H., Atchison C., Whitaker M., Ainslie K.E.C., Elliott J. Okell L., Redd R., Ashby D., Donnelly C.A., Barclay W., Darzy A., Cooke G., Riley S., Elliott P. (2020). Antibody prevalence for SARS-CoV-2 following the peak of the pandemic in England: REACT2 study in 100,000 adults. medRxiv https://doi.org/10.1101/2020.08.12.20173690. 
Figures

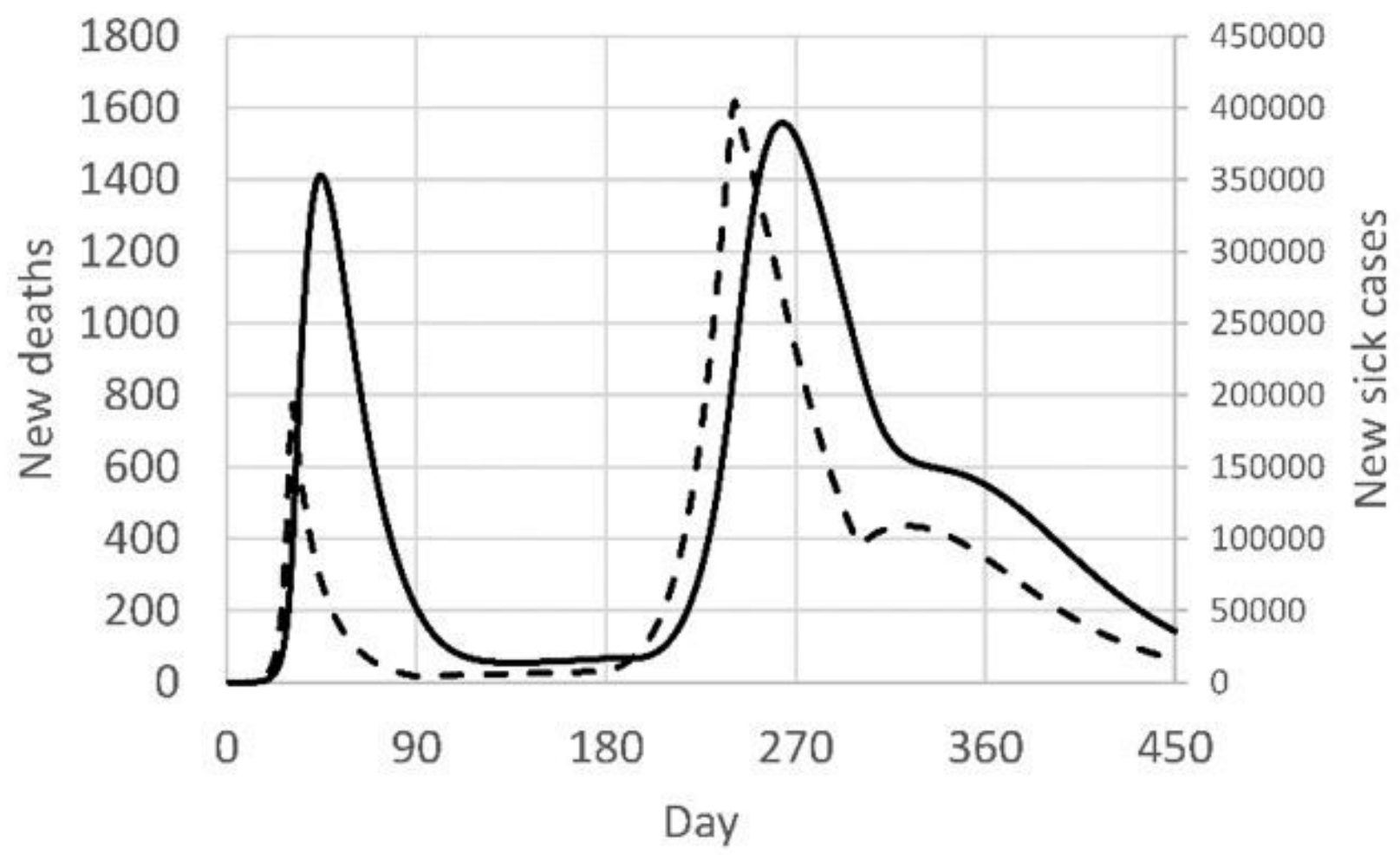

Figure 1

Daily new deaths (solid line, left axis) and new sick cases(dashed line, right axis) predicted by the model (extension 1) with the parameters from Table 2 


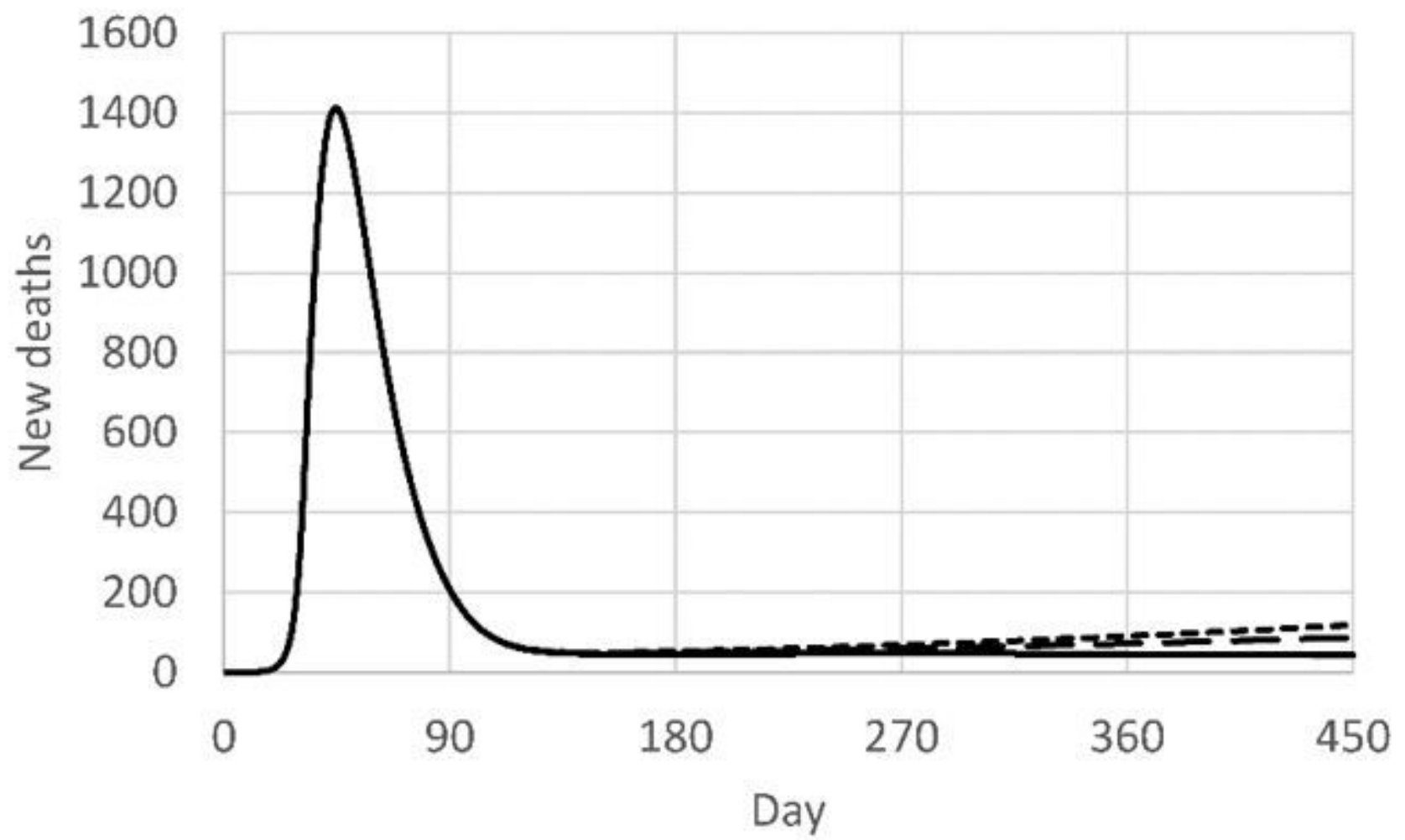

Figure 2

Effect of immunity loss on the onset of a second wave in a COVID-19 epidemic. Solid line: no loss of immunity; long dash: immunity disappears with a half-life of 6 months; short dash: immunity disappears with a half-life of 3 months. 


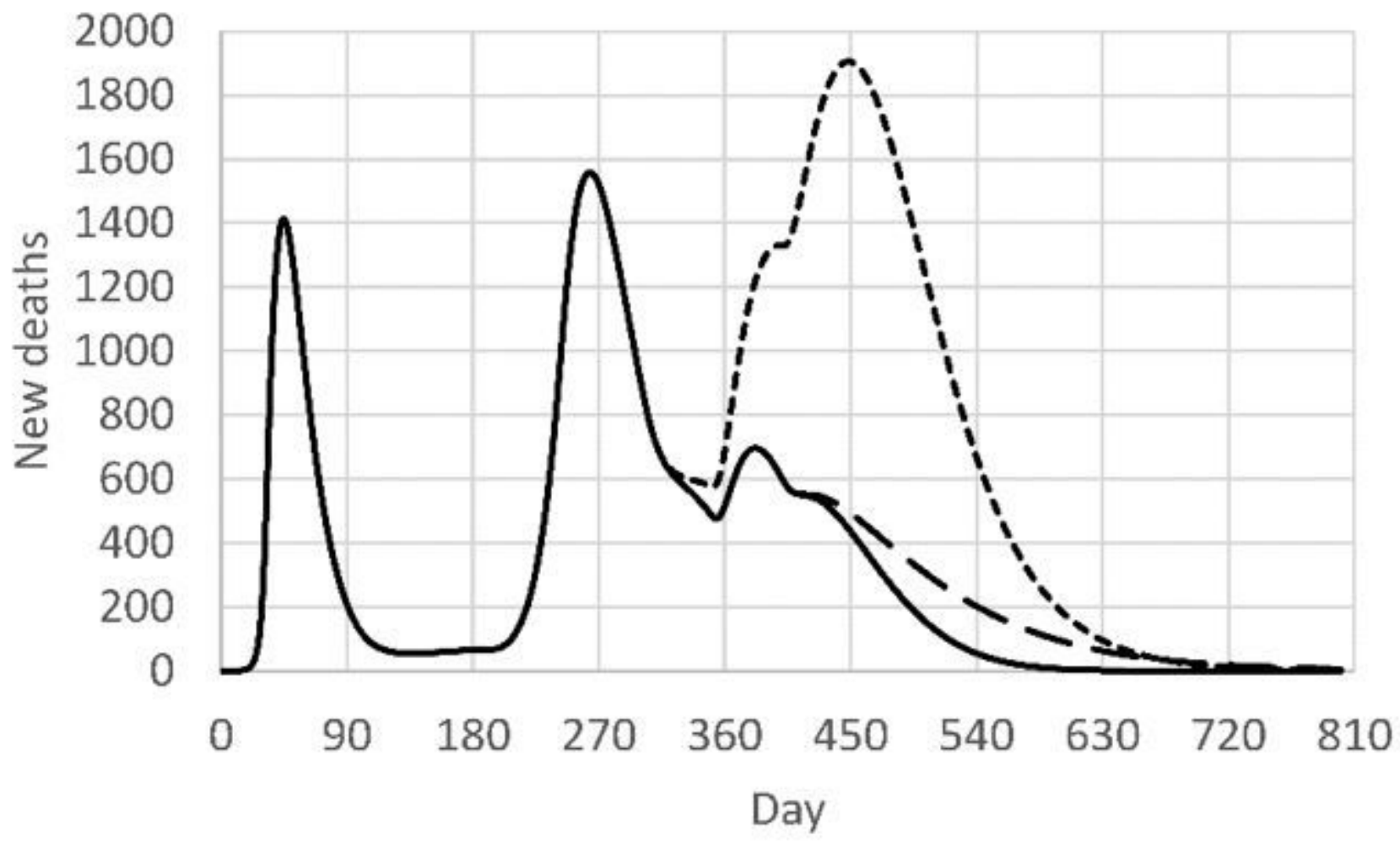

Figure 3

Daily deaths in three vaccination scenarios: school-going youth prioritized (day 300-400) followed by active population prioritized (day 400-500) (solid line); only school-going youth prioritized (day 300-400) (long dash); no vaccination (short dash). 


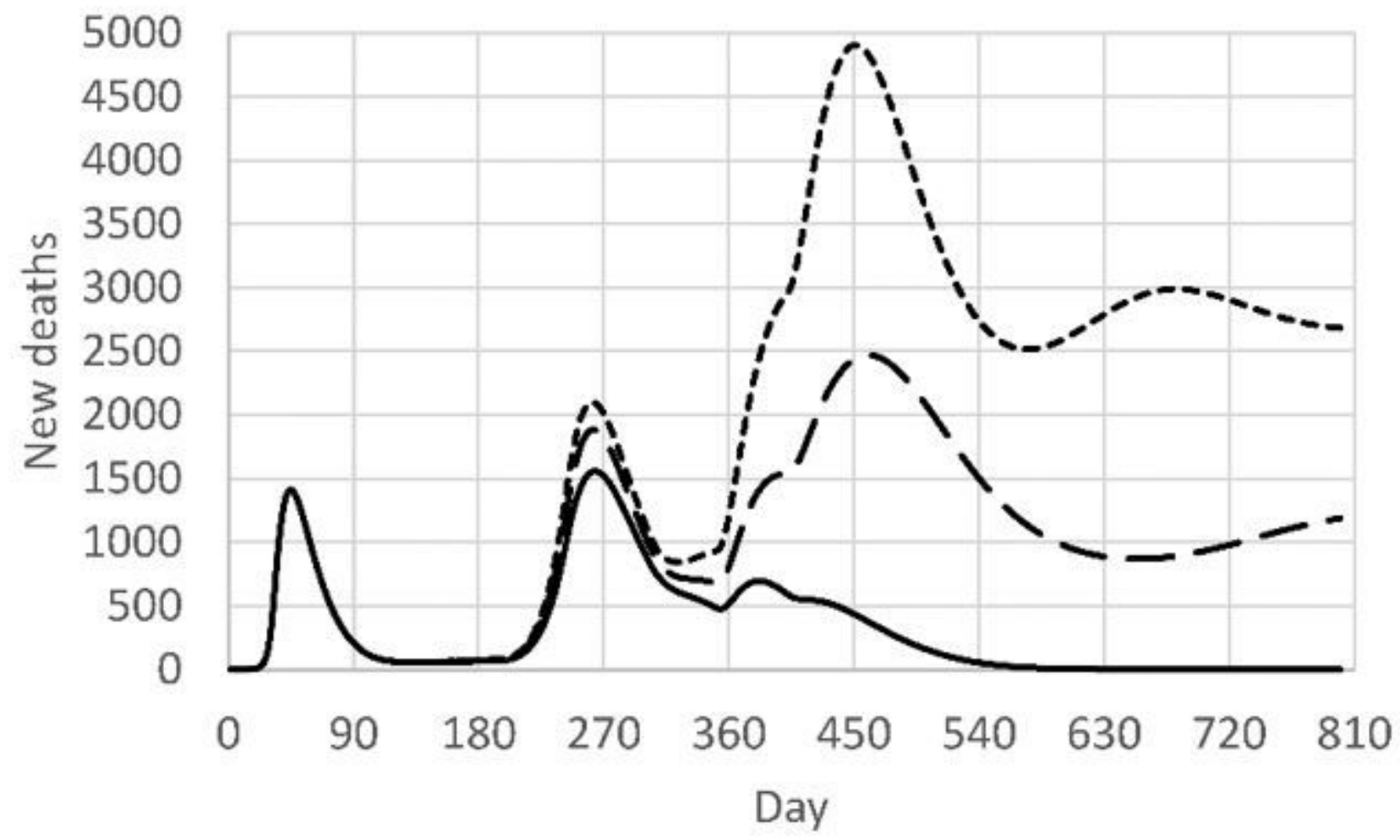

Figure 4

Daily deaths in continuing vaccination scenario (200,000 vaccines per day): no loss of immunity (solid line); immunity half-life 6 months (day 300-400) (long dash); immunity half-life 3 months (short dash). 


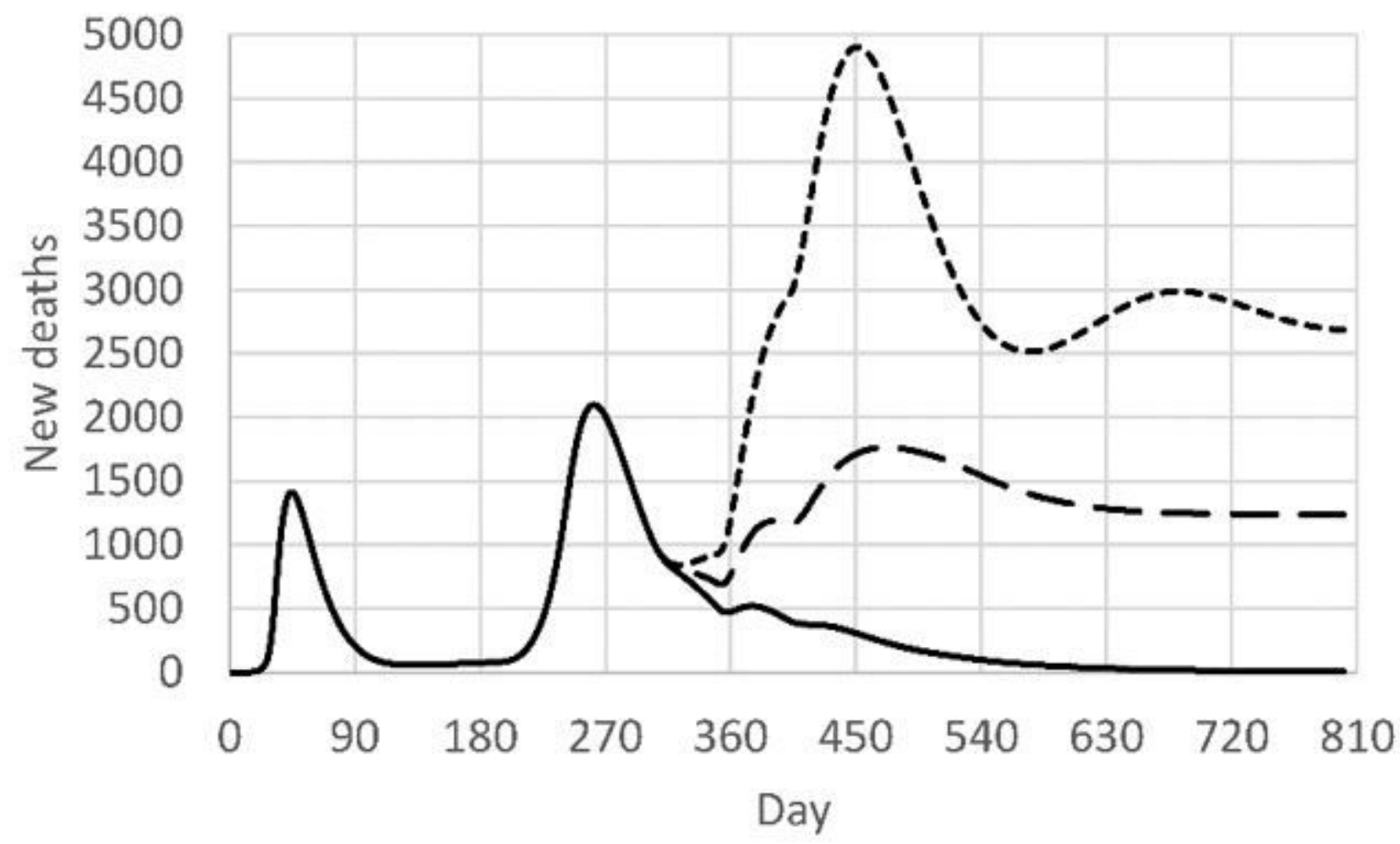

Figure 5

Daily deaths in continuing vaccination scenario, immunity half-life 3 months: 1,200,000 vaccines per day (solid line); 600,000 vaccines per day (long dash); 200,000 vaccines per day (short dash). 


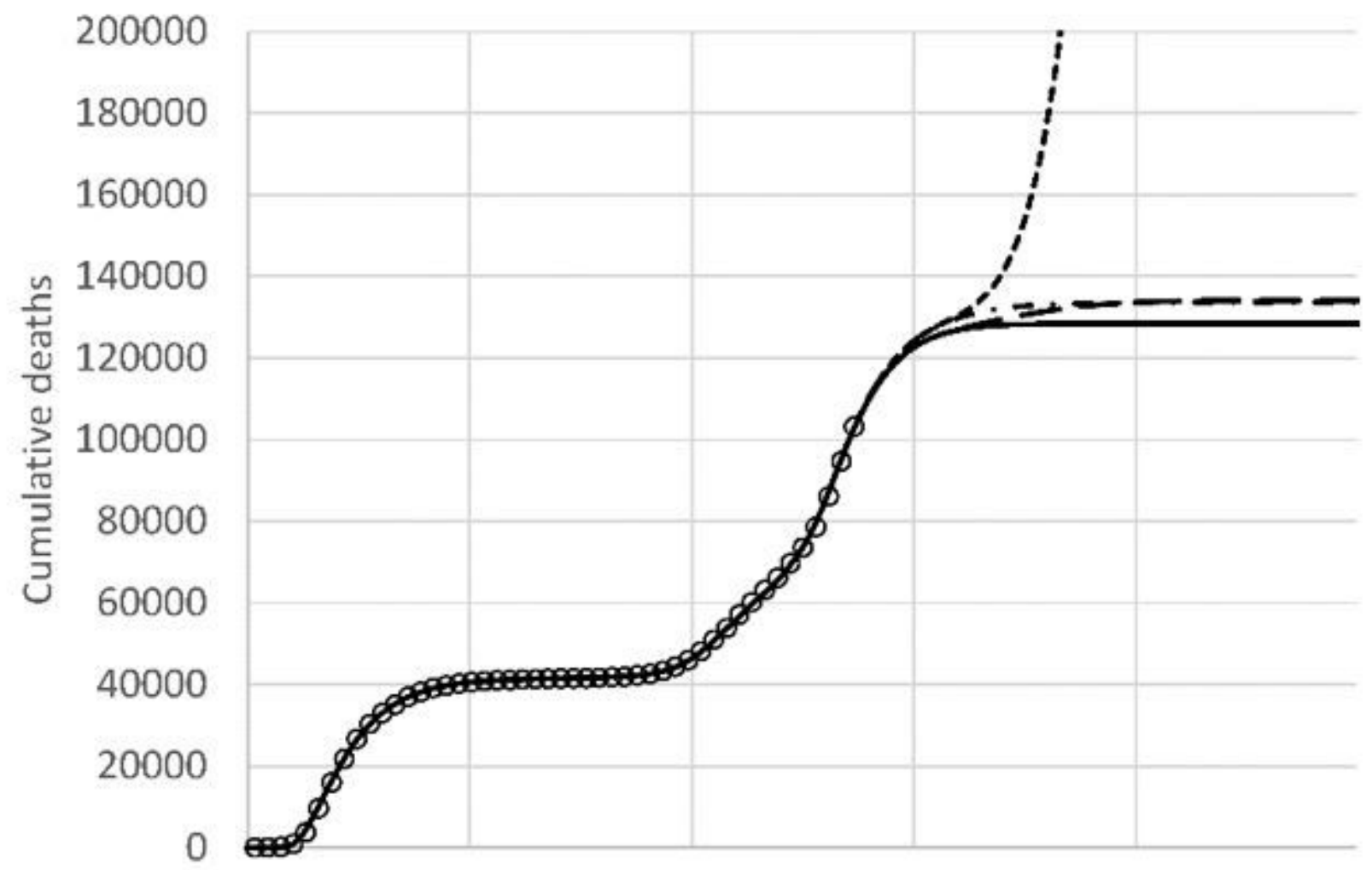

1-Mar-2020 1-Jul-2020 31-Oct-20202-Mar-2021 2-Jul-2021

- deaths reported — base case _ - - No vacc no NPI

- - vacc NPI -15\% ----n no vacc NPI -15\%

\section{Figure 6}

Cumulative deaths reported and modeled with vaccination, without loosening of NPIs (solid line); without vaccination, without loosening of NPIs (dot-dash line); with vaccination, with a $15 \%$ reduction of NPI effectiveness (long dash); and without vaccination, with a $15 \%$ reduction of NPI effectiveness (short dash). 


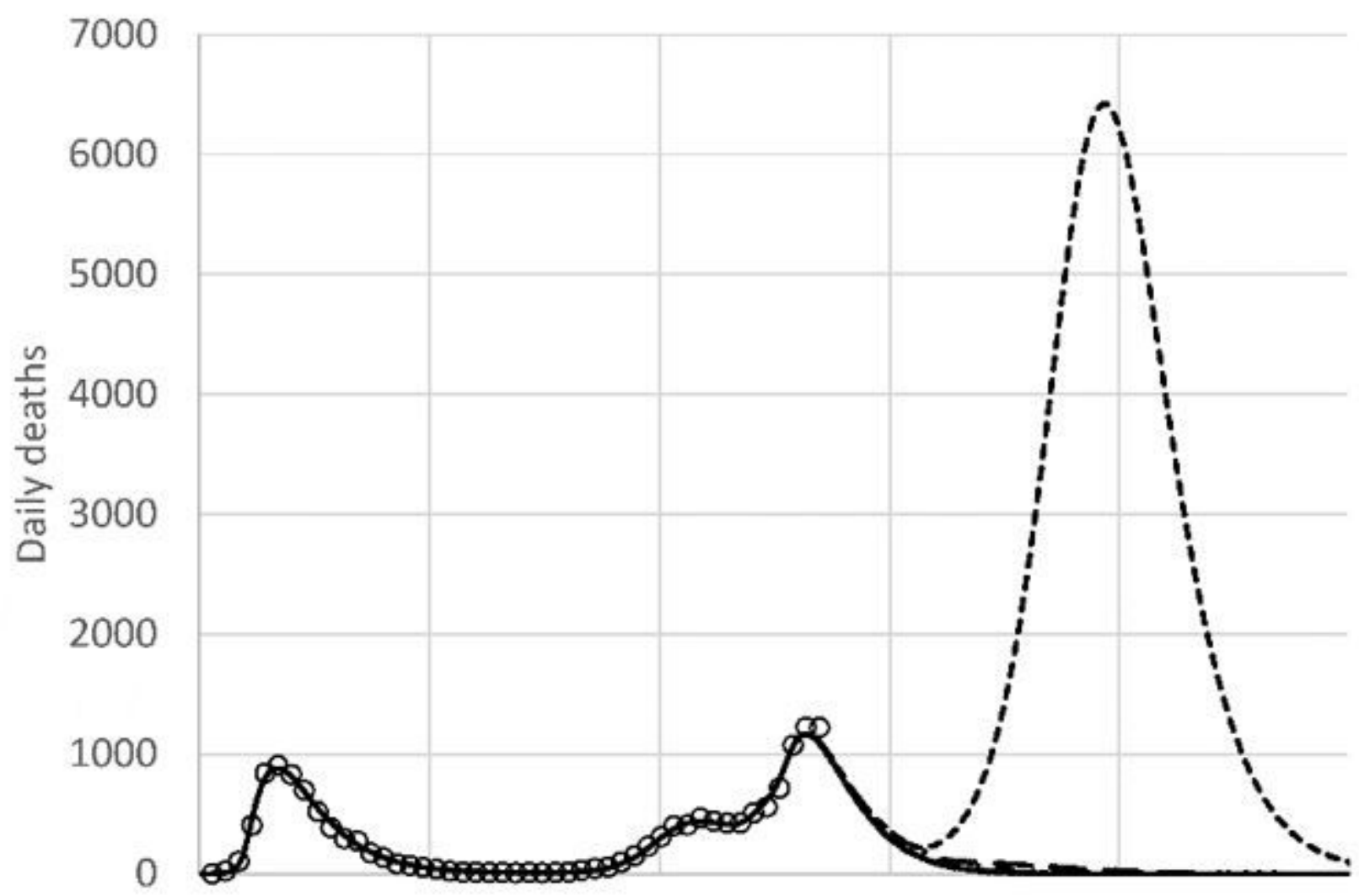

1-Mar-2020 1-Jul-2020 31-Oct-2020 2-Mar-2021 2-Jul-2021

- deaths reported — base case _- - No vacc no NPI

- - vacc NPI -15\% ----- no vacc NPI -15\%

Figure 7

Daily deaths reported (weekly averages) and modeled with vaccination, without loosening of NPIs (solid line); without vaccination, without loosening of NPIs (dot-dash line); with vaccination, with a $15 \%$ reduction of NPI effectiveness (long dash); and without vaccination, with a $15 \%$ reduction of NPI effectiveness (short dash). 


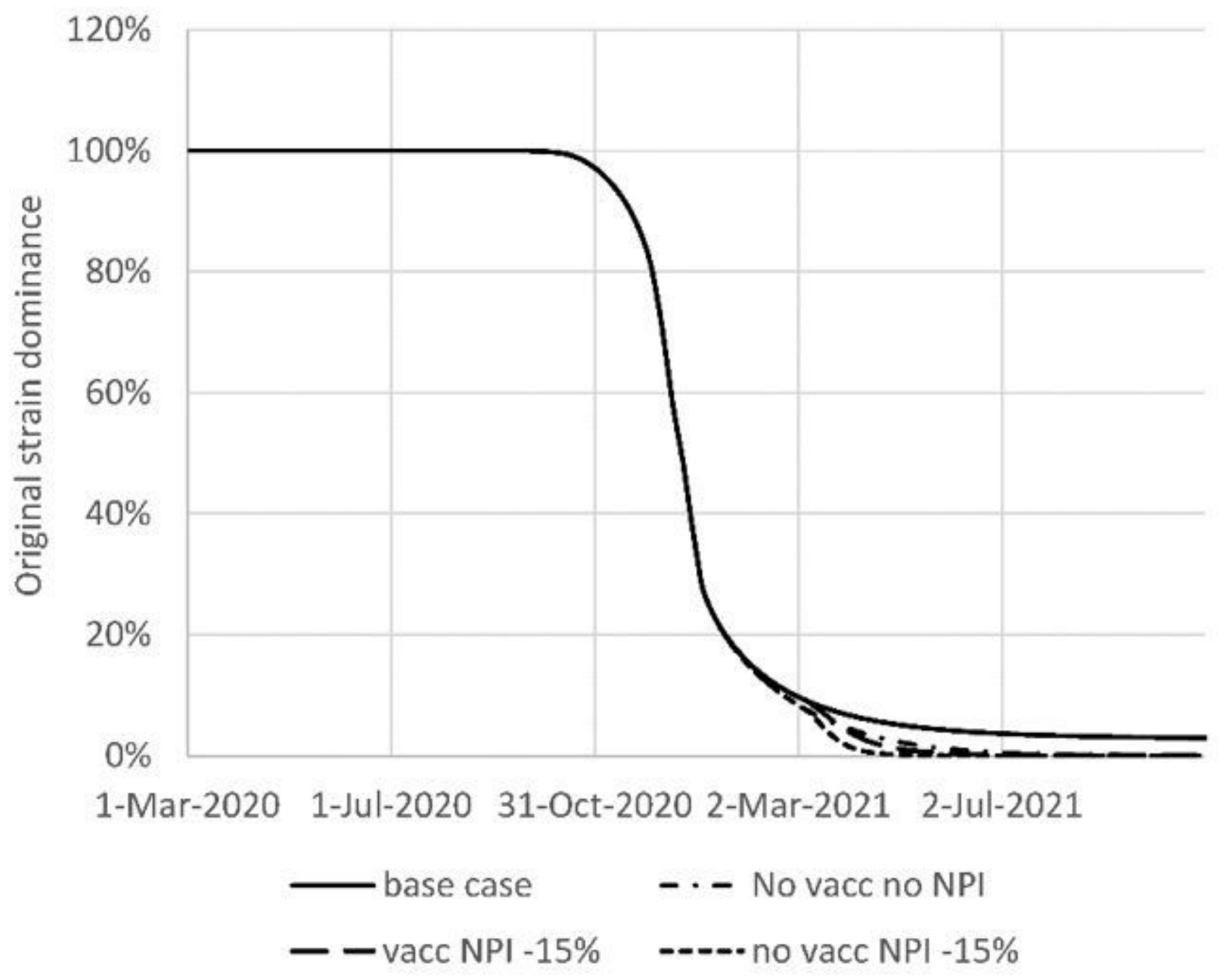

Figure 8

Dominance of the original (pre-September 2020) COVID-19 strains in the U.K. , as calculated by the model with the four scenarios from Figures 6 and 7. 